\title{
Fatigue behavior and cyclic damage of peek short fiber reinforced composites
}

\author{
A. Avanzini *, G. Donzella, D. Gallina, S. Pandini, C. Petrogalli \\ Mechanical and Industrial Engineering Department, University of Brescia, Via Branze 38, 25128 Brescia, Italy
}

\section{A R T I C L E I N F O}

\section{Article history:}

Received 10 February 2012

Received in revised form 25 May 2012

Accepted 8 June 2012

Available online 19 June 2012

\section{Keywords:}

A. Discontinuous reinforcement

B. Fatigue

C. Damage mechanics

\begin{abstract}
A B S T R A C T
Fatigue strength and failure mechanisms of short fiber reinforced (SFR) PEEK have been investigated in the past by several research groups. However some relevant aspects of the fatigue behavior of these materials, like cyclic creep and fatigue damage accumulation and modeling, have not been studied yet, in particular in presence of both fillers and short fibers as reinforcement. In the present research these aspects were considered by carrying out uni-axial fatigue tests in load control (cycle ratio $R=0$ ) on neat PEEK and PEEK based composites reinforced either with short carbon fibers only or with addition of fillers (graphite and PTFE). For each material stress-life curves were obtained and compared. Fatigue fracture surfaces were analyzed to identify failure mechanisms in presence of different reinforcement types. The evolution of cyclic creep strain was also monitored as a function of the number of cycles, thus allowing investigation on the correlation between cyclic creep parameters and fatigue life. The evolution of cyclic damage with loading cycles was then compared by defining a damage parameter related to the specimen stiffness reduction observed during the tests. Progressive cyclic damage evolution of short fiber reinforced PEEK composites presented significantly different patterns depending on applied stress level and on the presence of different reinforcement typologies. In order to reproduce the different fatigue damage kinetics and stages of progressive damage accumulation observed experimentally, a cyclic damage model was finally developed and implemented into a finite element code by which a satisfactory agreement between numerical prediction and experimental data at different stress levels for each examined material.
\end{abstract}

(c) 2012 Elsevier Ltd. All rights reserved.

\section{Introduction}

Efforts to lighten structures for weight sensitive applications have led to a growing use of short fiber reinforced (SFR) thermoplastics for structural components, taking advantage of the ease of processing and the lower costs traditionally associated with thermoplastics manufacturing processes. An ideal candidate to serve as a matrix for this class of composite materials is polyetheretherketone (PEEK) which exhibits excellent mechanical properties, even under continuous service conditions at elevated temperatures, in addition to good chemical resistance in many environments. PEEK mechanical properties can be further enhanced by adding special fillers to the short fiber reinforcement phase for specific purposes.

Failure mechanisms of SFR PEEK were investigated in the past [1-3], but fatigue behavior cannot be considered as completely understood, as in general for thermoplastic composites [4-6]. Effects of processing variables on fatigue in molded PEEK and its short fiber composites were discussed in $[7,8]$ considering fatigue crack propagation (FCP) aspects. Fatigue crack resistance showed

\footnotetext{
* Corresponding author. Tel.: +39 030 3715526; fax: +39 0303702448 .

E-mail address: andrea.avanzini@ing.unibs.it (A. Avanzini).
}

a complex interdependence of various matrix related factors such as molecular weight, crystallinity and fiber type, length, aspect ratio and preferred orientation. FCP has also been correlated to the microstructure by means of microstructural efficiency parameter related to the layered fiber distribution usually observed in SFR thermoplastics [9]. The effects of different load ratios and levels, specimen thickness and environmental temperatures were considered in [10-12]. Fatigue behavior of SFR thermoplastics has also been studied by a classical approach based on stress-life curves $(S-N)$. Rotating-bending fatigue tests on neat and short carbonfiber reinforced PEEK were carried out to investigate the fatigue characteristics of plain and notched specimens at room temperature [13]. More recently life curves were determined for PEEK reinforced with hydroxyapatite for orthopedic implants [14].

Cyclic creep aspects can be instead very significant if $S-N$ life curves are determined by carrying out uni-axial fatigue tests on standard tensile test specimens and tests are run in zero to tension loading (to avoid risk of specimen buckling), thus resulting in nonzero mean stress. This in turn may cause cyclic creep, since total strain may grow continuously with cycles number until final failure. Quite interestingly for SFR thermoplastics it has been suggested that fatigue life is somehow controlled by this cumulative strain [6] and the correlation between cyclic creep speed and the number of cycles to failure $\left(N_{\mathrm{f}}\right)$ in some cases has been reported 
to be stronger than that between maximum applied stress $\left(\sigma_{\max }\right)$ and $N_{\mathrm{f}}[16]$.

Overall the amount of available $S-N$ data for PEEK and its short fibers composites is limited, at least compared to FCP studies, and the role of cumulative strain and, for SFR PEEK based composites, the correlation between cyclic creep rate and $N_{\mathrm{f}}$ has not been studied yet.

On the other hand progressive degradation of material properties under cyclic loading condition may lead to premature component failure or inability to fulfill stiffness requirements in practical engineering applications. It is therefore obviously important to carefully evaluate cyclic damage and material properties evolution. Fatigue damage evolution in fiber reinforced composite materials is a quite complex phenomenon, especially in terms of onset and evolution. For some SFR thermoplastic, like polyamide reinforced with short glass fibers [17], fatigue damage evolution under cyclic loading has been found to occur according to different stages, involving initial matrix micro-cracks and matrix micro-voids formation, coalescence and propagation of defects and finally macroscopic crack propagation and fiber fracture. At a macroscopical level each stage resulted into different stiffness reduction rates as a function of cycle number. However for composite materials micro-mechanisms of damage accumulation may occur sometimes independently and interactively and the predominance of one and other of them may be strongly affected by both material variables and testing conditions [15]. As a result different patterns and stages of progressive damage evolution may be present depending on stress level, reinforcement phase and matrix but this kind of investigation has not yet been carried out on SFR PEEK.

In the present study, in order to investigate some of the above mentioned issues related to fatigue behavior and cyclic damage for this class of materials, an experimental campaign consisting of uni-axial fatigue tests on three different PEEK based materials (neat PEEK and PEEK reinforced with short carbon fibers in different amounts and fillers like PTFE and graphite) was carried out. Fatigue behavior of neat and reinforced matrix was compared in terms of $S-N$ curves and by analyzing fatigue fracture surfaces to identify different failure mechanisms.

The evolution of cyclic creep strain was also monitored as a function of the number of cycles, thus allowing investigation on the correlation between cyclic creep parameters and fatigue life. Progressive damage evolution of different materials was then considered by defining a damage parameter based on elastic modulus reduction to describe specimen stiffness reduction observed during the tests. Since fatigue damage investigation involves expensive and time consuming experimental campaigns, in particular when heterogeneous materials like short fiber reinforced composites are considered, it is also obviously important to develop fatigue damage models. Ideally these models should be versatile as much as possible to represent different patterns of cyclic damage evolution and not too complex in terms of material parameters determination, in order to reduce experimental effort needed for practical engineering application. For this reason in parallel to experimental testing a damage model was developed, basing on existing model proposed for SFR polyamides [17], to simulate damage evolution observed experimentally at a phenomenological level. Material parameters were determined for each examined material. Damage model was implemented into commercial FE code Abaqus ${ }^{\circledR}$ by customizing user subroutine USDFLD.

\section{Experimental methods}

\subsection{Materials and specimen}

Three different PEEK based materials were examined that will be referred in the text to as:
(1) Neat (unfilled grade PEEK, density $1.298 \mathrm{~g} / \mathrm{cm}^{3}$ ).

(2) C10-PVX (PEEK filled with 10\% wt. carbon micro-fibers, graphite and PTFE, density $1.445 \mathrm{~g} / \mathrm{cm}^{3}$ ).

(3) C30 (PEEK filled with 30\% wt. carbon micro-fibers, density $\left.1.411 \mathrm{~g} / \mathrm{cm}^{3}\right)$.

Neat PEEK is of interest for a variety of applications and was also studied as a reference for comparison purposes. C10-PVX is mainly employed in applications in which tribological aspects are of interest due to action of PTFE and graphite as internal lubricants. In particular graphite improves frictional behavior whereas the incorporation of PTFE reduces the friction coefficient and improves load carrying capacity under sliding condition $[18,19]$. C30 is representative of a class of PEEK composites of potential interest for structural applications.

Static and fatigue tests were carried out on dog-bone shaped specimens (Fig. 1) obtained from semi-finished extruded plates available commercially. For neat PEEK and C10-PVX the specimens were cut parallel to extrusion direction directly from plates having the desired thickness ( $6 \mathrm{~mm}$ ). For $\mathrm{C} 30$, due to manufacturing constraints on the dimensions of the semi-finished plate from which the specimens were taken (plate thickness $20 \mathrm{~mm}$ ), the specimens were obtained by cutting slices $6 \mathrm{~mm}$ thick on a plane perpendicular to the plate planar surface and then machining them to the dog-bone shape.

It should be noted that injection or extrusion molded SFR thermoplastics, such as the PEEK based composites under investigation, are structurally complex materials whose microstructure is set up during the manufacturing process. In particular for injection and compression molded SFR PEEK $[8,9,20]$ it has been reported the presence of a layered skin-core structure with different preferred fiber orientation, in which thickness and preferred layered orientations were dependent on processing conditions (flow speed, viscosity, temperatures). Specimens or industrial components made of materials with the same reinforcement but obtained under different processing/manufacturing conditions or cut under different directions may therefore exhibit significantly different microstructure and properties.

Such a kind of microstructure, if present, may significantly affect the material behavior. For this reason this aspect was investigated for each of the reinforced materials, even if in the present study samples were taken from semi-finished industrial products for which processing parameters were not disclosed and thus correlations between processing conditions and material properties could not be evaluated.

Some selected specimens were preliminary cut along longitudinal and transverse direction with respect to the specimen axis. After polishing cut surfaces were examined at the optical microscope (partially reproduced in Fig. $2 a$ and b). Overall for both C10-PVX and C30 the fiber distribution in the gage region of the specimen tested did not present a layered skin-core distribution, although it should be noted that C30 specimens were obtained by a machining operation that may be not completely representative of original layered structure. For C10-PVX, in which a lower

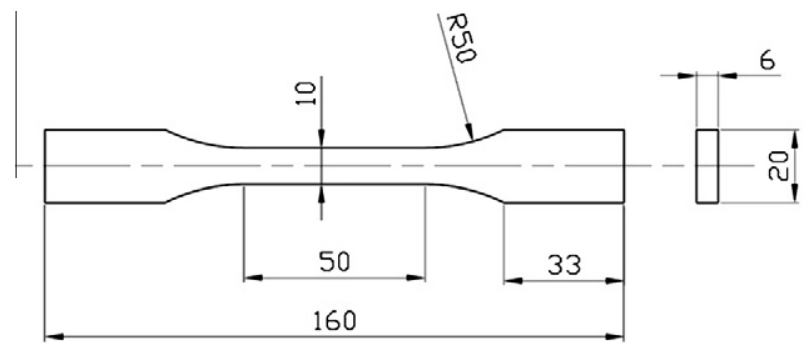

Fig. 1. Dog-bone specimen. 

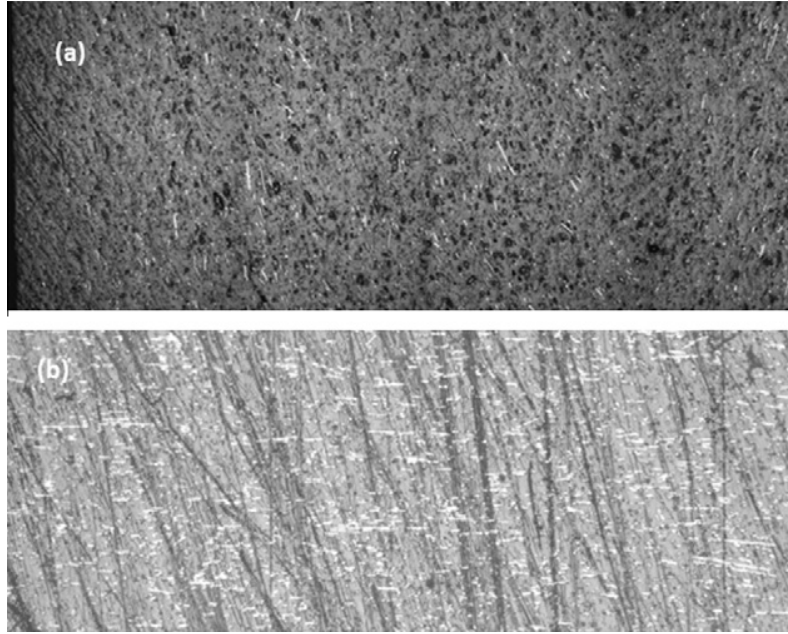

Fig. 2. Specimen section (partial) observed at optical microscope (a) C10-PVX, (b) C30.

amount of fiber is present and fillers were added, the material exhibited a highly heterogeneous microstructure and the distribution of fibers was not found to exhibit a distinct preferred orientation and the material. For C30 fibers appeared instead to be preferentially aligned with specimen axis.

\subsection{Test procedure}

Load-controlled tension-tension fatigue tests were performed on servo-hydraulic testing machine INSTRON 8501, in the range of cycles to failure from $10^{3}$ to $10^{6}$. Sinusoidal load cycles were applied keeping the load ratio $R=(\min$. load $) /(\max$. load $)=0$. The cyclic frequency was set to $10 \mathrm{~Hz}$. The applied nominal stress was evaluated by dividing the applied load by the reference section area. For each material 10-12 specimen were tested imposing a nominal maximum stress $\left(\sigma_{\max }\right)$ in the range between static tensile strength $\left(\sigma_{\mathrm{R}}\right)$ and run-out stress level.

Strain in the axial loading direction was measured by means of extensometer. Maximum and minimum strain values were recorded for each loading cycle whereas full stress-strain cycles were recorded at fixed time intervals, to allow observation of cyclic creep and damage evolution. For SFR composites the response to cyclic loading is characterized by hysteresis loops resulting in self-heating during fatigue tests. Temperature increase related to hysteresis and cyclic loading is therefore usually observed, as a function of loading frequency, imposed stress level, material properties and specimen geometry.

While mechanical properties of PEEK based materials undergo slight changes even at high temperatures, in order to ascertain that temperature increase was not excessive and to detect conditions under which failure occur for thermal instability, during the test temperature on the surface of the specimen was monitored with an infrared pyrometer.

Preliminary static tensile tests were carried out on specimens taken from the same batch used for fatigue test, at a cross-head speed of $0.25 \mathrm{~mm} / \mathrm{min}$, measuring strain with an extensometer (gage length $25 \mathrm{~mm}$ ).

\section{Results and discussion}

\subsection{Static tensile tests}

A summary of representative static tensile properties is given in Table 1. Results reported for static behavior show for C10-PVX a
Table 1

Mechanical properties from static preliminary tests.

\begin{tabular}{llcl}
\hline Material & $\begin{array}{l}\text { Elongation to rupture } \\
(\%)\end{array}$ & $\begin{array}{l}\text { Elastic modulus } \\
(\mathrm{MPa})\end{array}$ & $\begin{array}{l}\text { Tensile strength } \\
(\mathrm{MPa})\end{array}$ \\
\hline Neat & 15.5 & 3825 & 102 \\
C10- & 1.8 & 4368 & 60 \\
PVX & & 18600 & 190 \\
C30 & 1.65 & & \\
\hline
\end{tabular}

very limited increase of elastic modulus compared to neat PEEK, associated with a much more significant decrease of static strength, compared to unreinforced material.

On the contrary for C30 a higher fiber amount and degree of preferred alignment result in a significant increase of both elastic modulus and static strength (which is nearly doubled compared to neat PEEK).

Basing on these results we can conclude that fiber reinforcement effect is less effective for C10-PVX due to lower amount of fiber combined with a detrimental effect of particles added for lubrication purposes on material strength.

\subsection{Fatigue tests}

\subsubsection{Fatigue $S-N$ curves}

Fatigue life curves $(S-N)$ for examined materials are reported in Fig. 3 , in which maximum applied fatigue stress $\left(\sigma_{\max }\right)$ is plotted
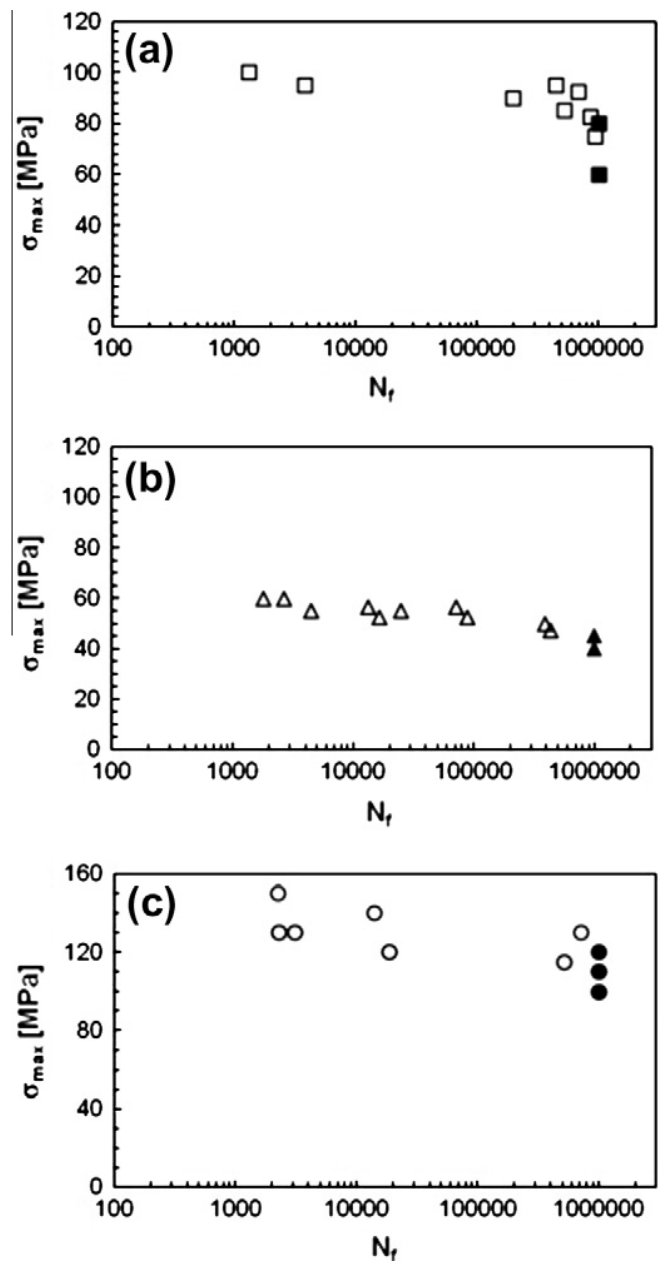

Fig. 3. Results of fatigue test (a) neat PEEK, (b) C10-PVX, (c) C30. 
vs. number of cycles to failure $\left(N_{\mathrm{f}}\right)$. Test results include run-out (i.e. test stopped after $10^{6}$ cycles). Considering neat PEEK specimens tested at values of maximum applied stress closer to tensile static strength did not reach thermal equilibrium and failed by thermal degradation due to excessive heating. Specimens tested at lower stress level reached thermal equilibrium with an increase of temperature of $5-20^{\circ} \mathrm{C}$ with respect to initial room temperature and exhibited a mechanically dominated type of failure. Overall fatigue data obtained in the present work are in line with findings reported in [13] which refer to rotating-bending fatigue tests carried out on cylindrical bars, with neat PEEK specimens able to withstand $10^{6}$ loading cycles at stress level up to about $70 \%$ of static strength.

Considering C10-PVX for specimens tested at maximum applied stress closer to tensile static strength temperature did not stabilize and increased up to $25^{\circ} \mathrm{C}$ at the time of failure. However even at maximum stress levels no thermal failure by degradation was observed by visual inspection. At lower stress levels C10-PVX reached thermal equilibrium with an increase of temperature ranging from $5{ }^{\circ} \mathrm{C}$ to $15^{\circ} \mathrm{C}$ with respect to initial room temperature. Compared to neat PEEK the fatigue behavior of CF10-PVX was very different, both considering maximum applied stress, which is about $40 \%$ lower, both regarding the shape of $S-N$ curve which in this case is rather flat with CF10-PVX specimens able to withstand $10^{6}$ loading cycles at stress level up to about $90 \%$ of static strength.

Considering C30 for specimens tested at maximum applied stress closer to tensile static strength temperature did not stabilize and increased up to $40^{\circ} \mathrm{C}$ at the time of failure. As for CF10-PVX even at maximum stress levels no thermal failure by melting was observed. At lower stress levels C30 reached thermal equilibrium with an increase of temperature ranging from $5{ }^{\circ} \mathrm{C}$ to $15{ }^{\circ} \mathrm{C}$ with respect to initial room temperature. Fatigue data confirmed good fatigue properties of SFR PEEK with C30 specimens able to withstand $10^{6}$ loading cycles at stress level up to about $60 \%$ of static strength, although stress-life curve in this case is more scattered with respect to those of the other materials.

Overall when considering fatigue behavior the main result is that fatigue strength of C30 is significantly higher than for neat PEEK and nearly double than that of C10-PVX, in line with results of static tests. Different values of fatigue/static strength ratio were also observed for C10-PVX and C30. This may suggest that different failure mechanisms could be present, but a direct comparison is not straightforward, since the materials were tested at rather different stress ranges. However some interesting observations on the role of reinforcing fiber phase on fatigue initiation and propagation failure processes for PEEK can be found in [13]. It was observed that fiber reinforcement give rise to a negative action against crack initiation, since micro-fibers can act as preferential crack nucleation points, and a positive action against crack propagation, since micro-fibers oppose crack propagation by dissipating energy in different ways (pull-out, fracture and debonding). In particular for CFR PEEK it was found that fatigue crack initiated from near the fiber ends due to stress concentration effects but crack growth rate was much less than that in the neat matrix. Similar reasoning could apply for the C30 PEEK investigated in the present study.

When considering C10 PVX the different fatigue strength and damage curves observed could be related to the different balance between these counteracting effects, due to the presence of PTFE and graphite and to the lower amount of fibers. The introduction of internal lubricants with low adhesion properties at the interface with the matrix results in a material which is even more full of "impurities" that can act as further preferential crack nucleation points with a lower amount of fibers acting as obstacles to the crack propagation.

\subsubsection{Fatigue fracture surfaces}

Fatigue fracture surfaces of specimens that failed at different stress levels were observed at SEM and optical microscope.

Considering neat PEEK at high stress level, the temperature increase led to melting of the specimens in the failure region. At lower stress level, when a mechanically dominated type of failure could be observed, fatigue fracture surface showed an irregular aspect with presence of regions having a mirror-like aspect. Previous studies on fatigue crack propagation [12] revealed that neat PEEK fatigue fracture is associated with the formation of different striation patterns on the fracture surface. Also the presence of parabolic or dimpled regions of micro-ductility has been reported, under both monotonic and cyclic loading conditions [7]. They are considered to be formed by the intersection of major crack front with secondary cracks nucleated ahead in the plastic zone. Quite interestingly, as shown in Fig. 4 in which a detail of a characteristic neat PEEK fracture surface is reported, both of the above mentioned features could be observed (indicated by arrow and circle). This indicates that fatigue failure (when failure was not of thermal nature) is essentially a cyclic phenomenon, as emphasized by the presence of striations, which may however interact with monotonic fracture mechanisms (dimples).

For SFR composites the failure mechanism in fatigue is often considered to consist of different stages [6]. The mechanisms during breakdown of the composites include fiber-matrix separation along the interfaces of fibers oriented parallel to the crack, deformation and fracture of the matrix between fibers, fiber pull-out and fracture of fibers transverse to crack direction. More specifically for PEEK based composites similar local fracture mechanisms have been observed on failure surfaces in FCP tests [12]. In these tests the degree of plastic deformation in the matrix was found to be related to loading conditions, with static loading resulting in a greater amount of matrix deformation. The presence of area of different roughness was also reported to be detectable on the fracture surface reflecting layered structure.

Considering C10-PVX fatigue fracture surfaces of C10-PVX tested at different stress level were very similar when observed at a macroscopical level. The only (slight) difference observed was a tendency to present at low stress a fracture surface lying in a single plane perpendicular to loading direction whereas at higher stress fracture surface is less planar and consist of multiple neighboring surfaces. Fracture surfaces presented a rather heterogeneous and irregular aspect, with presence of areas in which some of the above mentioned failure mechanisms above could be noticed, such as fiber/matrix separations along the interfaces of fibers

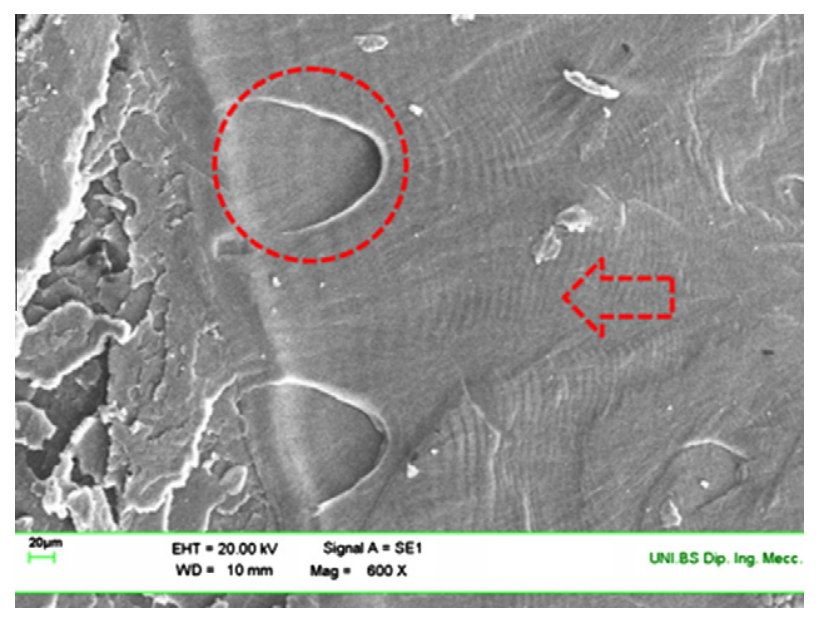

Fig. 4. SEM details of fatigue fracture surface of neat PEEK. 
oriented parallel to the fracture surface, and in some cases fiber breakage and pull-out (Fig. 5), as well as cavities and regions with different degrees of matrix deformation.

Considering C30 (Fig. 6) the higher fiber content and degree of orientation along the specimen axis resulted in fracture surfaces in which predominant fracture process appears to be of brittle nature, with a prevalence of fiber pull-out and fracture of fibers parallel to loading axis, although locally fiber/matrix separations along the interfaces of fibers oriented parallel fracture surface plane could also be observed.

\subsubsection{Cyclic creep}

In tension-tension fatigue tests mean value of strain may progressively shift along the strain axis, as qualitatively shown in Fig. 7, resulting in a progressive accumulation of strain. By continuously recording cycle by cycle maximum and minimum strain ( $\varepsilon_{\max }$ and $\varepsilon_{\min }$ respectively) and averaging their values, cyclic mean strain $\varepsilon_{\mathrm{m}}$ can be calculated. Then, by plotting $\varepsilon_{\mathrm{m}}$ vs. $N$ (number of cycles) the effects of progressive accumulation of strain during fatigue can be evaluated. As qualitatively shown in Fig. 8, depending on material and stress level, different behaviors could be observed. In general the rate of strain accumulation tends to stabilize at low stress after a short initial stage in which it rapidly increases, in some cases increasing more rapidly when approaching failure. At a high stress level instead the accumulated strain grew at a con-

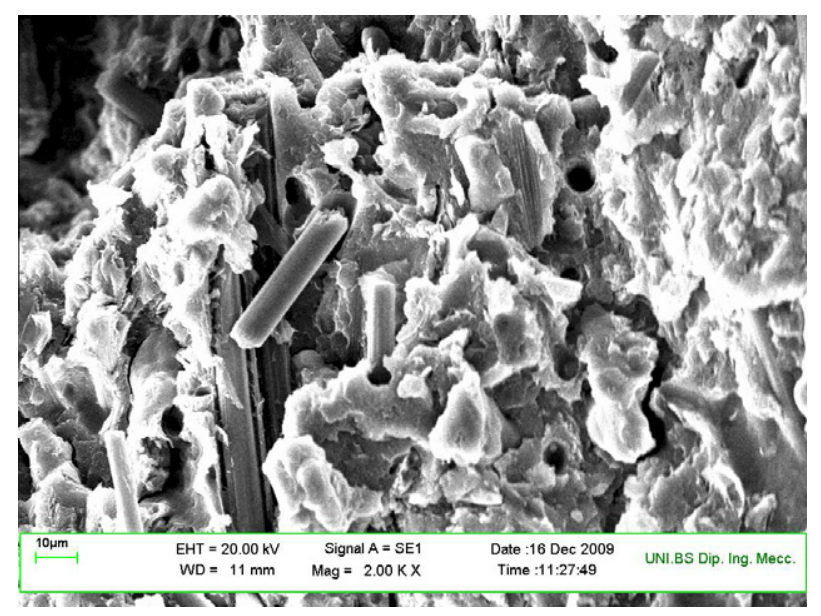

Fig. 5. SEM details of fatigue fracture surface of C10-PVX.

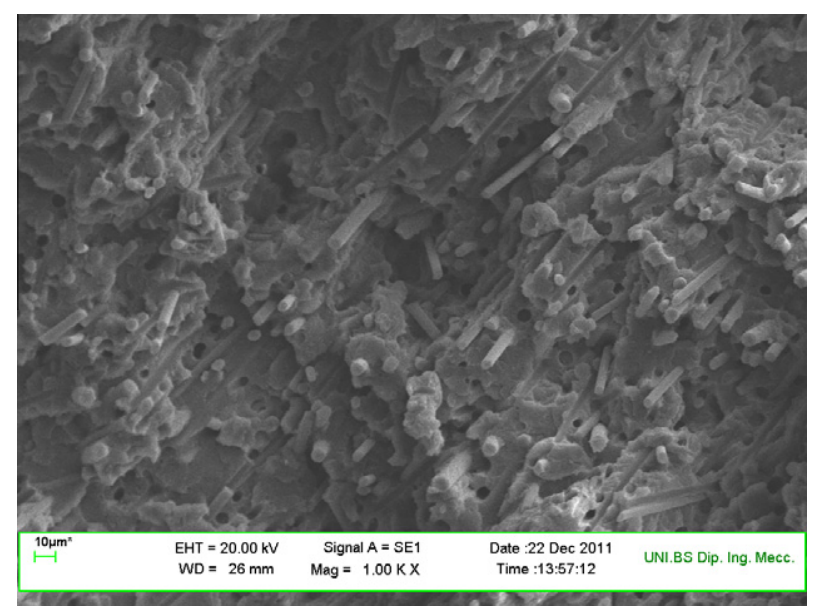

Fig. 6. SEM details of fatigue fracture surface of C30 PEEK.

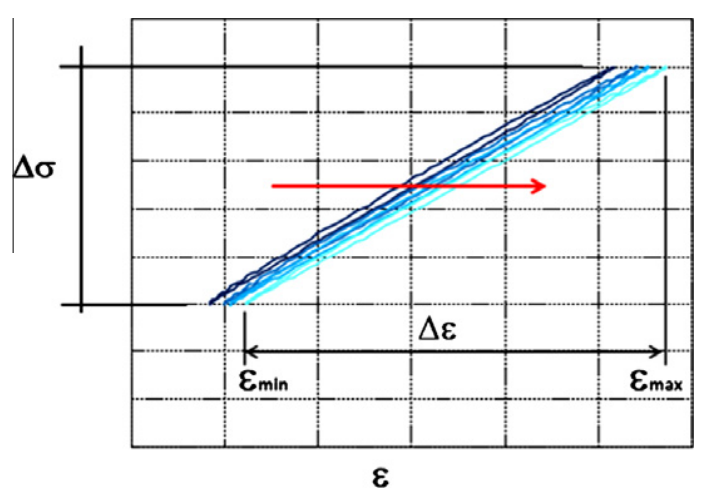

Fig. 7. Shift of stress-strain cycle and strain accumulation during fatigue tests.

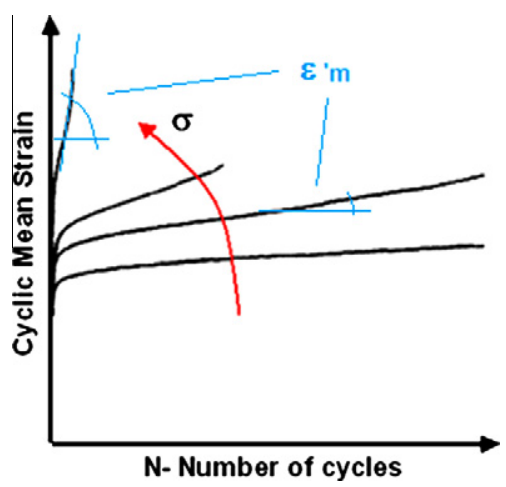

Fig. 8. Evolution of cyclic mean strain during fatigue tests.

stant and higher rate. This rate of accumulation can be described by a cyclic creep speed $\varepsilon_{\mathrm{m}}^{\prime}$, defined as:

$\varepsilon_{\mathrm{m}}^{\prime}=d \varepsilon_{m} / d N$

For examined materials cyclic creep speed $\varepsilon_{\mathrm{m}}^{\prime}$ observed during the tests was evaluated for each specimen by determining the slope of $\varepsilon_{\mathrm{m}}$ curve as a function of cycles $N$ either in the stabilized stage (when present) or, at high stress level, as a mean constant rate. Finally, in order to evaluate correlation between cyclic creep and fatigue failure of neat and SFR PEEK, $\varepsilon_{\mathrm{m}}^{\prime}$ was plotted as a function of $N_{\mathrm{f}}$ (Number of cycles to failure), as shown in Fig. 9.

Results obtained in the present work showed a significant correlation for C30 and to a slightly lesser extent for C10-PVX between cyclic creep and number of cycles to failure. This can be noticed by considering Fig. 9b and $\mathrm{c}$ where values of coefficient of determination $R^{2}$ for a linear fit on $\log \left(\varepsilon_{\mathrm{m}}^{\prime}\right)$ and $\log \left(N_{\mathrm{f}}\right)$ are close to unity for both materials. For neat PEEK some correlation also exists, however data appear to be more scattered and cyclic creep speed for specimens which failed by thermal degradation could be strongly influenced by temperature increase as well.

Similar experimental evidences were noticed for SFR polyamide 6 in [16], and present results, in particular for CF30, confirm that cyclic creep speed could be a significant parameter to evaluate fatigue behavior of this class of materials.

The significance of correlation between cyclic creep speed and fatigue lifetime for SFR composites was also discussed in [16]. It was inferred that a good correlation could be an indication of lower sensitivity to notches since variations in lifetimes of the specimens are mainly determined by global parameters, as creep is a phenomena of global character. For SFR composites this interpretation would be supported by the fact that SFR composites, contrary to unfilled polymers, are full of local notches due to the presence of 

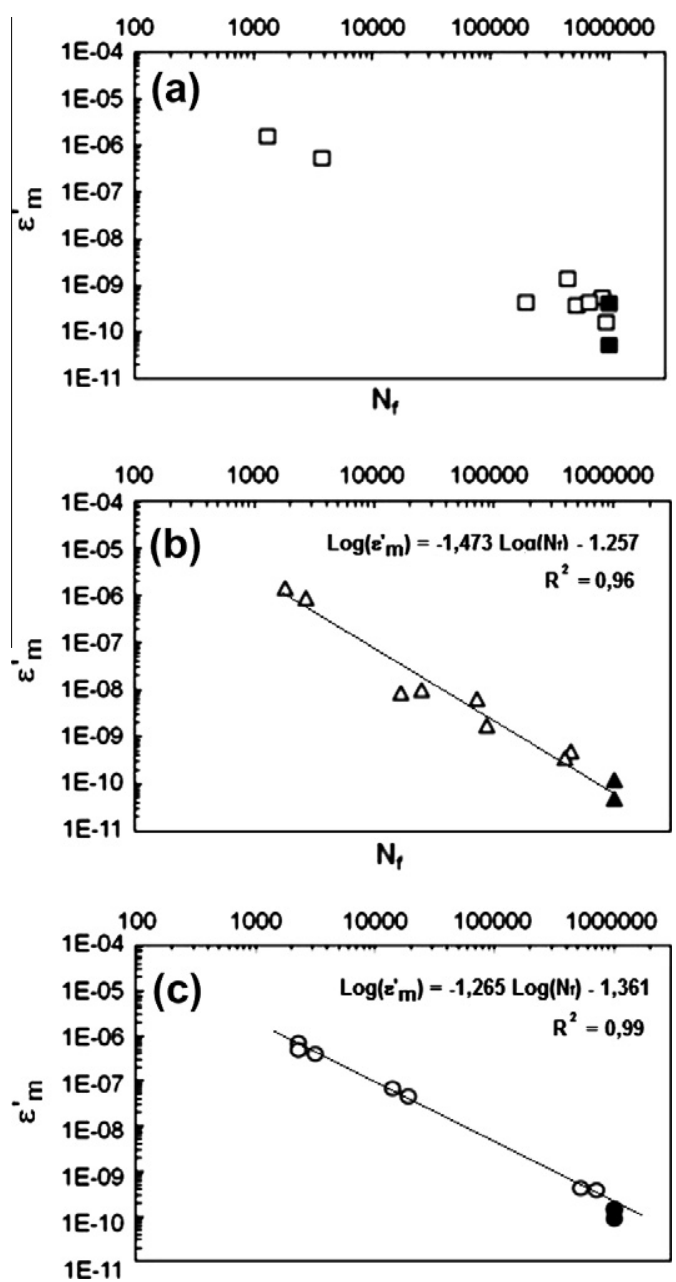

Fig. 9. Cyclic creep speed during fatigue tests (a) neat PEEK, (b) C10-PVX, (c) C30.

fibers. As a consequence, local variations (like scratches or notches) should have a lower influence on fatigue lifetime compared to the effects of collective notches (fiber ends) present in the material. Quite interestingly experimental comparison of the fatigue strength of plain and notched specimens of neat and short carbon fiber reinforced PEEK reported in literature [13], showed that unfilled material was more sensitive to notches than reinforced material.

Good correlation between cyclic creep speed and fatigue lifetime observed especially for CF30 is in line with these findings. However in order to support or modify this conclusion further investigations are needed to consider this aspect in more detail. In particular the fact that different slopes of the $\log \left(\varepsilon_{\mathrm{m}}^{\prime}\right)$ vs $\log \left(N_{\mathrm{f}}\right)$ curves were observed for C30 and C10-PVX, associated with a slightly higher correlation for C30 compared to C10-PVX (coefficient of determination $R^{2}=0.99$ and 0.96 respectively) suggests that different sensitivity to notches may be observed for different composite systems. Further investigations are planned to study the dependency of notch sensitivity on notch size and in presence of microstructures consisting either of fiber only or of fiber and fillers.

\subsubsection{Fatigue damage}

The fatigue damage behavior of neat PEEK and its SFR composites was studied by analyzing the evolution of the stress-strain cycle during the tests. In particular in load controlled tests as a result of material damage the amplitude of strain range $\Delta \varepsilon$ due to imposed and constant stress range $\Delta \sigma$ may tends to increase with increasing cycle number. This result at a macroscopical level in a reduction of specimen stiffness and the progressive modification of stress-strain loop can then simply described by defining a phenomenological damage index $D$ :

$D=1-\frac{E}{E_{0}}$

in which $E$ is the elastic modulus, defined as the ratio $\Delta \sigma / \Delta \varepsilon$, and $E_{0}$ is its value at the first cycle (see Fig. 7). The evolution of damage index $D$ as a function of the cycles number is represented for neat and SFR PEEK in Fig. 10. Examined materials presented different trends. For neat PEEK at low stress level damage rate is higher in the initial stage and then it tends to stabilize. Stiffness reduction due to excessive heating at high stress level is immediately recognizable by a sudden change of the slope of the damage curve as failure approaches. For C10-PVX $D-N$ curve slope increases with higher stress level, with a short initial stage in which damage index increased rapidly, followed by a prolonged second stage in which a lower and more constant slope can be observed. Even at low stress level and even if temperature reached a stable value, the damage index did not tend to stabilize completely, also for specimen that reached $10^{6}$ cycles. For C30 the stiffness reduction, as for C10-PVX, progressively increased with cycle number but with a different trend, due to the presence of a third stage in which slope of $D-N$ curve, after remaining constant, tends to increase as final failure approaches. Although $D$ is basically a phenomenological parameter and its experimental measurement is also influenced by temperature, the
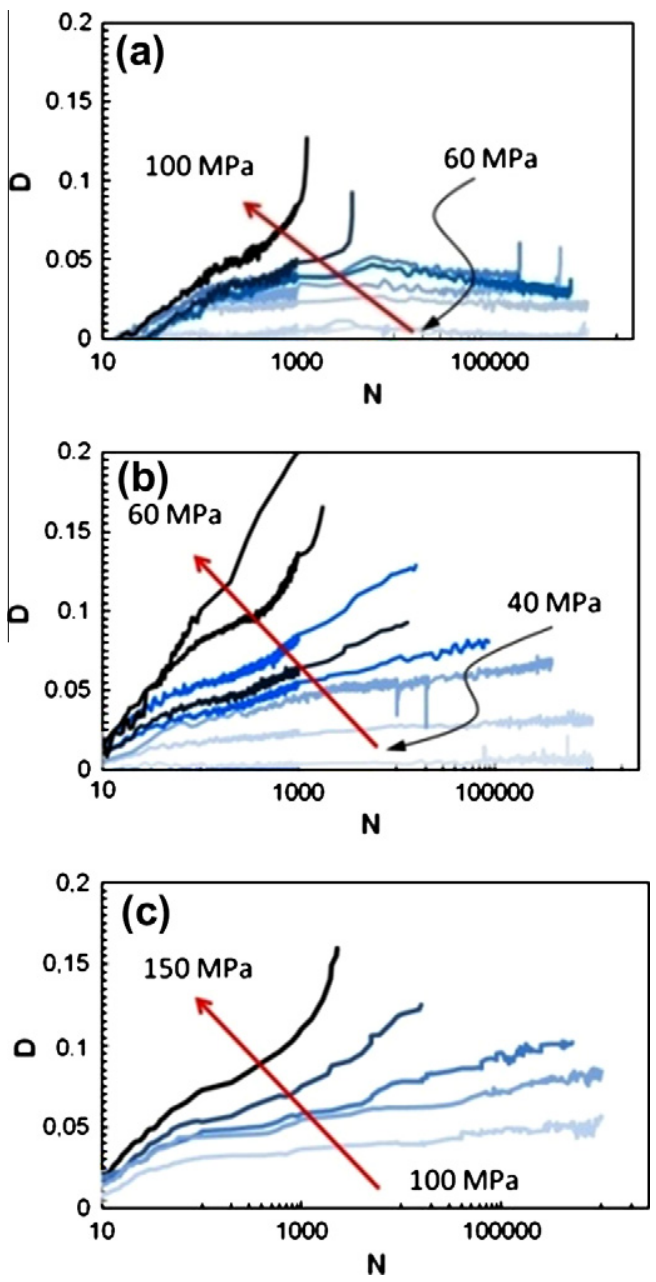

Fig. 10. Damage index during fatigue tests (a) neat PEEK, (b) C10-PVX, (c) C30 
differences observed between C10-PVX and C30 on a macroscopical level may help to understand different fatigue damage mechanisms that could be present in the different stages.

In particular in the pattern of damage evolution for C30, especially at higher stress levels, one can recognize the presence of regions with different rates of stiffness reductions. These are similar to those reported for materials like polyamide reinforced with short glass fibers, in which initial high stiffness reduction, possibly related to formation of matrix micro-cracks and micro-voids, is followed by a stage of more gradual stiffness reduction, due to coalescence and propagation of defects, leading to a final stage in which a fast stiffness reduction is again observed, associated with macroscopic crack propagation and fiber fracture.

This damage mechanisms chronology was also established using ultrasonic techniques (C-Scan) for fatigue damage and fatigue post-impact damage of short glass fiber reinforced composites [22].

For FC10-PVX instead variations of stiffness reduction rates are less evident, with initial stage being very rapid, if any, and a final stage of fast instable fracture growth hardly noticeable.

It should be noted however that considering the first stage of damage evolution, an initial reduction of elastic modulus could be detected for both neat and reinforced PEEK in similar extent, that suggesting that even if damage could exist in significant extent an important role is also played by the thermo-viscoelastic property of the matrix.

\subsection{Fatigue damage modeling}

\subsubsection{Background of modeling approach}

Upon a modeling point of view results confirmed the need for a versatile cyclic damage model, that must be capable to reproduce different trends and slope changes of $D-N$ curve depending on stress level applied or material considered. A possible approach for the prediction of cyclic damage evolution for SFR composites has been proposed by Meraghni [17]. In the following some key points of Meraghni's phenomenological approach will be recalled to help discussion on the application of such damage model to PEEK based composites, with some simplifying hypotheses, basing on the set of experimental data available. For a more detailed description of model theoretical background the reader may refer to $[17,21]$. With this approach damage is modeled under the assumption that response of the undamaged material is orthotropic linearly elastic and that damage is diffuse. The strain energy density function of the damaged material, denoted by $W_{d}$, is defined as a function of the strain tensor $\left(\varepsilon_{i j}\right)$ as):

$$
\begin{aligned}
2 W_{d}= & \frac{1}{1-v_{12} v_{21}}\left[E_{11}^{0}\left(1-d_{11}\right) \varepsilon_{11}\left\langle\varepsilon_{11}+v_{21} \varepsilon_{22}\right\rangle_{+}+E_{11}^{0} \varepsilon_{11}\left\langle\varepsilon_{11}+v_{21} \varepsilon_{22}\right\rangle_{-}\right] \\
& +\frac{1}{1-v_{12} v_{21}}\left[E_{22}^{0}\left(1-d_{22}\right) \varepsilon_{22}\left\langle\varepsilon_{22}+v_{12} \varepsilon_{11}\right\rangle_{+}+E_{22}^{0} \varepsilon_{22}\left\langle\varepsilon_{22}+v_{12} \varepsilon_{11}\right\rangle_{-}\right] \\
& +G_{12}^{0}\left(1-d_{12}\right) \gamma_{12}^{2}+G_{13}^{0}\left(1-d_{13}\right) \gamma_{12}^{2}+G_{23}^{0}\left(1-d_{23}\right) \gamma_{23}^{2}
\end{aligned}
$$

where $E_{i j}^{0}$ (with $i=j, 1,2$ ) and $G_{i j}^{0}$ (with $i \neq j, 1,2,3$ ) are the elastic moduli of the material in the undamaged state. Quantities $\langle A\rangle+$ and $<$ A $>-$ stand for the positive and negative parts of amount $A$, respectively and therefore, the damage affects the Young's moduli only when $\varepsilon_{11}+v_{21} \varepsilon_{22}$ is positive, with the underlying assumption that compressive loading doesn't affect the crack growth and damage evolution and consequently damage evolves only during tension loading. Damage is represented by $d_{i j}$, so that elastic properties of the damaged material are:

$$
\begin{aligned}
& E_{i j}=E_{i j}^{0}\left(1-d_{i j}\right) \\
& G_{i j}=G_{i j}^{0}\left(1-d_{i j}\right)
\end{aligned}
$$

Damage rates consist of a sum of two components: the first contribution is derived from a Norton-like power law and the second component is introduced to describe the stiffness reduction occurring during the first damage stage. Damage rates are then derived from a system of differential Eq. (6):

$\frac{\partial d_{i j}}{\partial N}=\dot{d}_{i j}=\frac{\alpha_{i j} \beta_{i j}}{1+\beta_{i j}}\left(Y_{i j}\right)^{\beta_{i j}-1}+\lambda_{i j}\left(Y_{i j}\right)\left(e^{-\left(\delta_{i j} N\right)}\right)$

where $\alpha, \beta, \lambda$ and $\delta$ are material damage constants, $N$ is the cycle number whereas $Y_{i j}$ are functions representing thermodynamic dual forces associated with damage rates and defined as per Eq. (7):

$Y_{i j}=-\frac{\partial W_{d}}{\partial d_{i j}}$

In this way, by integration of Eq. (6) cycle by cycle, it is possible to obtain damage variables $d_{i j}$ and then describe damage evolution as a function of cycle number $N$.

\subsubsection{Isotropic damage model for SFR PEEK}

In the present work a similar approach was adopted but introducing some modifications to take into account the characteristics of the materials examined and some limitations related to the test procedure and loading conditions.

In particular basing on the experimental data available (mainly uniaxial test) it was assumed to treat material damage as isotropic, since it was not possible, at this time, to carry out investigations on material properties in different directions or under different loading conditions. For C10-PVX this assumption is, at least partially, supported by optical and SEM microscope observations which did not reveal the presence of a clear short fibers preferred orientation. On the other hand for $\mathrm{C} 30$ a preferred alignment with specimen axis could be noticed and furthermore even if the damage is isotropic at the beginning (initiation) it has been demonstrated that for such composites materials there is an induced anisotropy due to the damage propagation and accumulation.

For these reasons the adopted modeling approach should be regarded as first attempt to evaluate the applicability of this kind of damage model to the examined materials, leaving to future work investigations on anisotropic nature of damage.

Under isotropy assumption the orthotropic model proposed by Meraghni could be simplified by considering only one damage state variable $d$ representing the stiffness reduction, during cyclic loading. In this way the elastic properties of the material in the damaged state are simply:

$E=E^{0}(1-d)$

$G=G^{0}(1-d)$

As a consequence strain energy for the damaged material may be written as per Eq. (10):

$$
\begin{aligned}
2 W_{d}= & \frac{E^{0}(1-d)}{1-v^{2}}\left[\varepsilon_{11}\left\langle\varepsilon_{11}+v \varepsilon_{22}\right\rangle_{+}+\varepsilon_{22}\left\langle\varepsilon_{22}+v \varepsilon_{11}\right\rangle_{+}\right] \\
& ++\frac{E^{0}}{1-v^{2}}\left[\varepsilon_{11}\left\langle\varepsilon_{11}+v \varepsilon_{22}\right\rangle_{-}+\varepsilon_{22}\left\langle\varepsilon_{22}+v \varepsilon_{11}\right\rangle_{-}\right] \\
& +\frac{E^{0}(1-d)}{2(1+v)} \gamma_{12}^{2}
\end{aligned}
$$

and definition of the thermodynamic dual variable $Y$ correspondingly reduces to Eq. (11):

$$
\begin{aligned}
Y= & \frac{E^{0}}{2\left(1-v^{2}\right)}\left[\varepsilon_{11}\left\langle\varepsilon_{11}+v \varepsilon_{22}\right\rangle_{+}+\varepsilon_{22}\left\langle\varepsilon_{22}+v \varepsilon_{11}\right\rangle_{+}\right] \\
& +\frac{E^{0}}{4(1+v)} \gamma_{12}^{2}
\end{aligned}
$$


A further modification stems from the stress controlled loading condition adopted experimentally. Having defined $Y$, in order to obtain the damage variable $d_{i j}$, for strain controlled tests it would possible to integrate directly the damage rate $\partial d / \partial N$ (Eq. (6)). However, for stress controlled tests, strain continuously changes and therefore direct integration of the damage rates $\partial d / \partial N$ is not possible [17]. It would then be necessary to integrate numerically over any loading cycle. Since a fatigue test may consist of thousands of cycles, this would bring about an exponential increase in the processing time needed for calculations. In order to avoid these time consuming integration problems, related to stress controlled tests, a direct definition of damage was assumed. In particular damage state variable $d$ able to correctly describe the damage evolution of SFR PEEK was defined as a function of non-dimensional value of $Y$ as in Eq. (12):

$d=\alpha_{\text {iso }} \ln (N) \cdot Y^{\beta_{\text {iso }}(1-Y)}+\delta_{\text {iso }} N Y$

in which coefficients $\alpha_{\text {iso, }}, \beta_{\text {iso, }}, \delta_{\text {iso }}$ are material damage constants. The logarithmic factor $\ln (N)$ describes the first stiffness reduction due to material softening and the second factor $Y^{\beta \text { iso(1-Y) }}$ is used to control the curves trend at different levels of applied stress in the second stage of the fatigue damage evolution curve. The last part of the equation $\left(\delta_{\text {iso }} N Y\right)$ controls the trend of the third stage of the damage evolution curve. Basing on experimental results a fitting procedure was applied to determine damage parameters to be introduced in the model to describe material damage. The equations defining the damage model previously described were implemented into an electronic calculation sheet. The fitting procedure was applied to experimental damage curves corresponding to different imposed stress levels and values of material damage constants were found to allow a satisfactory matching between experimental and analytical prediction.

It should be remarked that the identification procedure for the original Meraghni's model requires an inverse approach for identifying the whole set of independent damage parameters based on digital image correlation techniques [23].

The values of material damage constants for C10-PVX and C30 are reported in Table 2. Due to the differences in the pattern of damage evolution discussed in Section 3.2.3, for C10-PVX, only the first addend of the damage state variable $d$ Eq. (8) is present,

Table 2

Material damage constants.

\begin{tabular}{|c|c|c|c|c|c|}
\hline \multirow[t]{2}{*}{ Stress level } & \multicolumn{4}{|l|}{ C30 } & \multirow{2}{*}{$\begin{array}{l}\text { C10-PVX } \\
\text { All }\end{array}$} \\
\hline & $100 \mathrm{MPa}$ & $115 \mathrm{MPa}$ & $120 \mathrm{MPa}$ & $130 \mathrm{MPa}$ & \\
\hline$\alpha_{\text {iso }}$ & 0.0150 & 0.0160 & 0.0187 & 0.0190 & 0.0190 \\
\hline$\beta_{\text {iso }}$ & 1.70 & 1.70 & 1.70 & 1.70 & 1.77 \\
\hline$\delta_{\text {iso }}$ & 0 & 0 & $2.0 \times 10^{-6}$ & $1.7 \times 10^{-5}$ & - \\
\hline
\end{tabular}

since the third stage of its experimental damage evolution curve is negligible or not present and the values of $\alpha_{\text {iso }}$ and $\beta_{\text {iso }}$ were the same independently of stress level applied.

For C30 $\alpha_{\text {iso }}$ and $\beta_{\text {iso }}$ were found to be dependent on stress level and in order to correctly model the third stage of damage evolution also $\delta_{\text {iso }}$ coefficients were determined.

It should be noted that ideally the material parameters should have to be independent of displacement or load levels to guarantee a more general applicability of the model. This limitation probably arises from the previously discussed assumptions concerning application of Meraghni's model under isotropic conditions and from the direct definition of damage variable $d$, adopted for computational reasons. In this respect it would be of particular interest extending experimental investigation on the anisotropic nature of damage.

\subsubsection{Damage model numerical implementation}

Having defined damage variables and equations governing damage evolution as a function of strain field, the next step was the numerical implementation of the damage model into the finite element (FE) code Abaqus ${ }^{\circledR}$. By taking advantage of model formulation in the strain field this goal was achieved by customizing user subroutine USDFLD. This subroutine allows defining field variables at a material point as a function of time or of any available material point quantities and it can be used to introduce solutiondependent material properties defined as functions of field variables. In particular the damage model previously described was implemented in the code to simulate damage accumulation during tension-tension uni-axial fatigue tests. The FE model consists of a 3D dumb-bell specimen with dimensions and shape equal to real specimen. Boundary conditions consisted in clamping the specimen at an extremity and applying an axial cyclic load at the other one, with a load ratio $R=0$. The mesh used for the model was obtained from a convergence study and consisted of 136 elements with four nodes. The logical workflow for model implementation into FEM code was the following:

1. Run simulation by applying load to reproduce sinusoidal fatigue cycle.

2. Obtain strain field related to the maximum stress value reached during each fatigue cycle by means of USDFLD.

3. Evaluate the thermodynamic dual variable $Y$ and calculate the correspondent damage state variable $d$ by implementing in USDFLD Eqs. (11) and (12).

4. Pass values of damage variable back to FEM code and update material properties (according to Eqs. (8) and (9)) to be used in the next cycle by means of USDFLD.

5. Repeat step $1 \rightarrow 4$ till a set number of cycles or till a predefined limit value of the damage is reached.

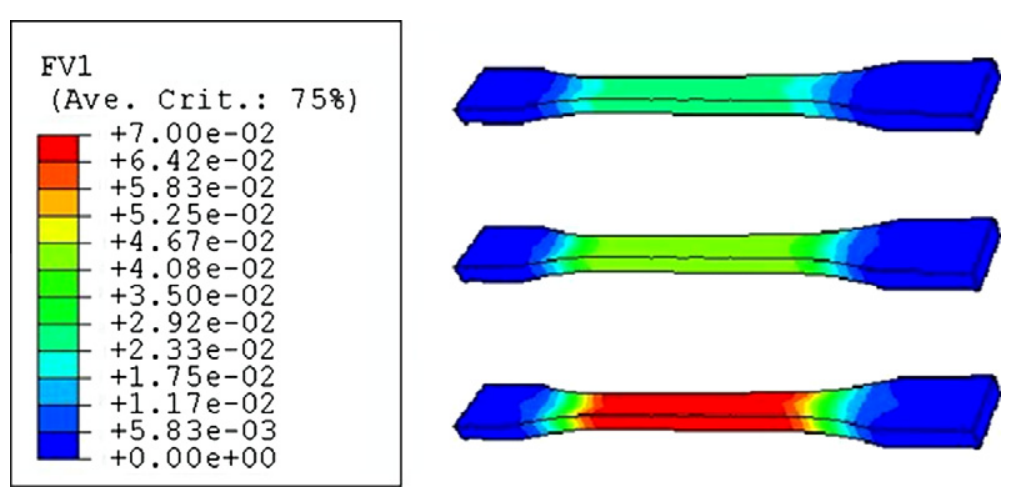

Fig. 11. Damage distribution in a C10-PVX specimen tested at $61 \mathrm{MPa}$ in load control. 

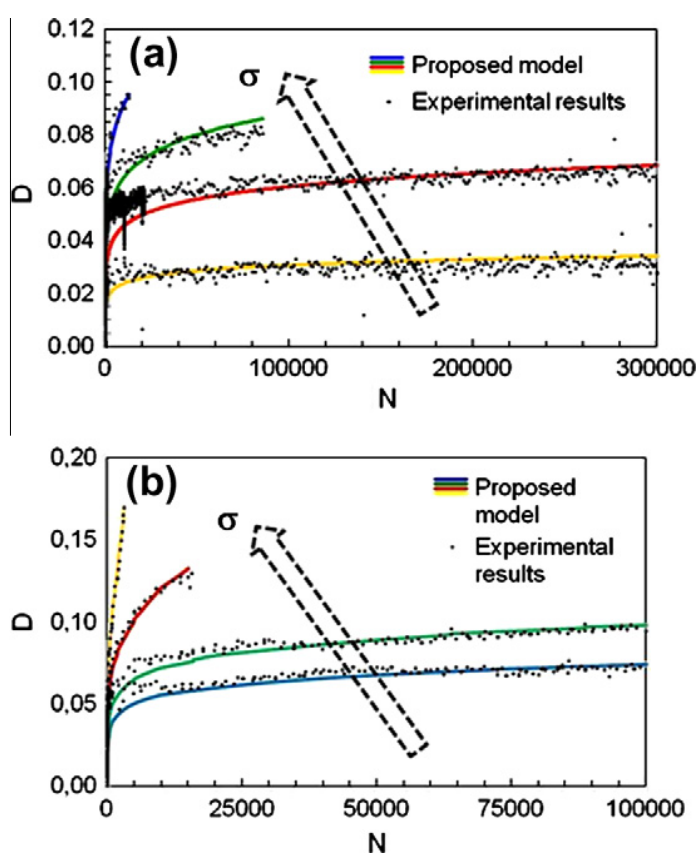

Fig. 12. Experimental and simulated behavior at selected different stress levels (a) C10-PVX, (b) C30.

The outputs of the FE analysis are the fatigue damage value $d$ (indicated as Field Value FV1) and the stress at each cycle expressed at the Gauss point. As an example the progressive evolution of predicted damage during the simulation of a test with a longitudinal applied stress equal to $61 \mathrm{MPa}$ is shown for C10-PVX in Fig. 10.

The damage levels and evolution predicted by means of the FEM damage model were compared, at different stress levels, with the actual damage evolution obtained from experimental tests, as shown in Figs. 11 and 12 for C10-PVX and C30 respectively. Despite simplifying assumption concerning the isotropic nature of PEEK composite studied and direct definition of damage variable as per Eq. (12), overall it may be observed that the numerical model allowed a satisfactory prediction of the damage evolution of C10-PVX and C30 at each of the different stress levels. The implementation of the model in a FEM code will allow the extension of this approach to the study of practical structural applications.

\section{Conclusions}

In the present study different aspects of fatigue behavior of neat PEEK and its short fiber composites were investigated by uni-axial fatigue tests. The effects on static and fatigue strength of PEEK reinforcement with short carbon fibers were found to be significantly dependent on the amount of fiber, with a marked increase of both properties for C30 compared to neat PEEK. Lower amount of fibers associated with presence of fillers to enhance tribological properties resulted instead in a decrease of both static and fatigue strength properties. A significant correlation between cyclic creep parameters and fatigue life was found, in particular for C30 PEEK indicating that cyclic creep speed could be a significant parameter to evaluate fatigue behavior of this class of materials. Microscope observation of fatigue fracture surface evidenced some differences between failure mechanisms of examined SFR PEEK which correspond, at a macroscopical level, to different trends of progressive damage evolution. Comparing fatigue damage the three materials presented different patterns of damage accumulation, depending on presence and characteristics of reinforcement phase. In particular in the pattern of damage evolution for C30, especially at higher stress levels, one can recognize the presence of regions with different rates of stiffness reductions. For C10-PVX instead variations of stiffness reduction rates are less evident, with initial stage being very rapid, if any, and a final stage of fast instable fracture growth hardly noticeable. Basing on experimental findings an isotropic fatigue damage accumulation model for SFR PEEK was proposed, taking as a reference some recent work of fatigue damage modeling for short fiber reinforced composites. The model, formulated in the strain space is based on the definition of damage variables as a function of thermodynamic dual forces. Material parameters to be introduced in the damage law were then determined to fit experimental data. Finally numerical implementation into a commercial FE code was carried out. The implementation of the damage model allowed predicting the fatigue damage evolution in a PEEK short fiber composite at different applied stress with good agreement between the damage predicted by the model and the experimental damage observed.

\section{Acknowledgements}

The paper describes some results of a research which has been carried out as a part of the project AMDACOMP, financed by the Italian University and Research Ministry PRIN2007 Grant, on the topic of damage analysis and monitoring in polymeric composite materials.

\section{References}

[1] Motz H, Schultz JM. Mechanical failure in PEEK and its short fiber-composites. J Thermoplastic Compos 1990;3:111-29.

[2] Wu G, Schultz JM. Processing, microstructure and failure behavior in shortfiber-reinforced Poly(Ether Ether Ketone) composites. Polym Compos 1990;11(2):126-32.

[3] Sarasua JR, Remiro PM, Pouyet J. The mechanical behavior of PEEK short fiber composites. J Mater Sci 1995;30(13):3501-8.

[4] Lang RW, Manson JA, Hertzberg RW. Mechanisms of fatigue fracture in short glass fiber-reinforced polymers. J Mater Sci 1987;22:4015-30.

[5] Mandell JF, McGarry FJ, Huang DD. Fatigue of glass and carbon fiber reinforced engineering thermoplastics. Polym Compos 1981;2(3):137-44.

[6] Horst JJ, Spoormaker JL. Mechanisms of fatigue in short glass fiber reinforced polyamide 6. Polym Eng Sci 1996;36(22):2718-26.

[7] Saib KS, Isaac DH, Evans WJ. Effects of processing variables on fatigue in molded PEEK and its short fiber composites. Mater Manuf Processes 1994;9(5):829-50.

[8] Saib KS, Isaac DH, Evans WJ. The effect of short carbon fiber reinforcement on fatigue crack growth in PEEK. Composites A 1996;27A:547-54.

[9] Friedrich K, Walter R, Voss H, Karger-Kocsis J. Effect of short fiber reinforcement on the fatigue crack propagation and fracture of PEEK-matrix composites. Composites 1986;17:205-16.

[10] Brillhart M. Fatigue fracture behavior of PEEK. J Reinf Plast Compos 1993;12:943-50.

[11] Brillhart M, Gregory BL, Botsis J. Fatigue fracture behaviour of PEEK. I. Effects of load level. Polymer 1991;32(9):1605-11.

[12] Brillhart M, Botsis J. Fatigue fracture behaviour of PEEK. II. Effects of thickness and temperature. Polymer 1992;33(24):5225-32.

[13] Nisitani H, Noguchi H, Kim YH. Evaluation of fatigue strength of plain and notched specimens of short carbon-fiber reinforced polyetheretherketone in comparison with polyetheretherketone. Eng Fract Mech 1992;43(5):685-705.

[14] Abu Bakar MS et al. Tensile properties, tension-tension fatigue and biological response of polyetheretherketone-hydroxyapatite composites for loadbearing orthopedic implants. Biomaterials 2003;24:2245-50.

[15] Mao H, Mahadevan S. Fatigue damage modelling of composite materials. Compos Struct 2002;58:405-10.

[16] Horst JJ, Spoormaker JL. Fatigue fracture mechanisms and fractography of short-glassfiber-reinforced polyamide 6. J Mater Sci 1997;32:3641-51.

[17] Nouri H, Meraghni F, Lory P. Fatigue damage model for injection-molded short glass fiber reinforced thermoplastics. Int J Fatigue 2009;31:934-42.

[18] Friedrich K, Ahang Z, Schlarb AK. Effects of various fillers on the sliding wear of polymer composites. Compos Sci Technol 2005;65:2329-43.

[19] Inami $S$ et al. Effect of fine-dispersed PTFE on load carrying capacity of PEEK. Lubr Sci 2008;20:299-310.

[20] Lee DJ. Comparison of mechanical properties of compression and injection molded PEEK/Carbon fiber reinforced composites. Key Eng Mater 2006;306308:751-6. 
[21] Ladeveze P, Le Dantec E. Damage modelling of the elementary ply for laminated composites. Compos Sci Technol 1992;43:257-67.

[22] Margueres Ph, Meraghni F, Benzeggagh ML. Comparison of stiffness measurements and damage investigation techniques for a fatigued and postimpact fatigued GFRP composite obtained by RTM process. Compos A Appl Sci Manuf 2000;31(2):151-63.
[23] Meraghni F et al. Parameters identification of fatigue damage model for short glass fiber reinforced polyamide (PA6-GF30) using digital image correlation. Procedia Eng 2011;10:2110-6. 